\title{
Family conferences and shared prioritisation to improve patient safety in the frail elderly (COFRAIL): study protocol of a cluster randomised intervention trial in primary care
}

Achim Mortsiefer ${ }^{1 *} \mathbb{0}$, Stefan Wilm¹, Sara Santos', Susanne Löscher ${ }^{1}$, Anja Wollny², Eva Drewelow², Manuela Ritzke ${ }^{2}$, Petra Thürmann ${ }^{3}$, Nina-Kristin Mann ${ }^{3}$, Gabriele Meyer ${ }^{4}$, Jens Abraham ${ }^{4}$, Andrea Icks ${ }^{5}$, Joseph Montalbo ${ }^{5}$, Birgitt Wiese ${ }^{6}$, Attila Altiner ${ }^{2}$ and on behalf of the COFRAIL study group

\begin{abstract}
Background: Frailty in elderly patients is associated with an increased risk of poor health outcomes, including falls, delirium, malnutrition, hospitalisation, and mortality. Because polypharmacy is recognised as a possible major contributor to the pathogenesis of geriatric frailty, reducing inappropriate medication exposure is supposed to be a promising approach to improve health-related quality of life and prevent adverse outcomes. A major challenge for the process of deprescribing of inappropriate polypharmacy is to improve the communication between general practitioner (GPs), patient and family carer. This study investigates the effects of a complex intervention in frail elderly patients with polypharmacy living at home.

Methods: This is a cluster randomised controlled trial including 136 GPs and 676 patients. Patients with a positive clinical screening for frailty are eligible if they are aged 70 years or older, receiving family or professional nursing care at home, and taking in five or more drugs per day. Exclusion criteria are higher grade of dementia and life expectancy of 6 months or less. The GPs of the intervention group receive an educational training promoting a deprescribing guideline and providing information on how to conduct a family conference focussing on prioritisation of treatment goals concerning drug therapy. During the 1-year intervention, GPs are expected to perform a total of three family conferences, each including a structured medication review with patients and their family carers. GPs of the control group will receive no training and will deliver care as usual. Geriatric assessment of all patients will be performed by study nurses during home visits at baseline and after 6 and 12 months. The primary outcome is the hospitalisation rate during the observation period of 12 months. Secondary outcomes are number and appropriateness of medications, mobility, weakness, cognition, depressive disorder, healthrelated quality of life, activities of daily living, weight, and costs of health care use.

(Continued on next page)
\end{abstract}

* Correspondence: achim.mortsiefer@med.uni-duesseldorf.de

'Institute of General Practice, Medical Faculty, Heinrich-Heine-University, Moorenstr. 5, 40225 Düsseldorf, Germany

Full list of author information is available at the end of the article

C The Author(s). 2020 Open Access This article is licensed under a Creative Commons Attribution 4.0 International License, which permits use, sharing, adaptation, distribution and reproduction in any medium or format, as long as you give appropriate credit to the original author(s) and the source, provide a link to the Creative Commons licence, and indicate if changes were made. The images or other third party material in this article are included in the article's Creative Commons licence, unless indicated otherwise in a credit line to the material. If material is not included in the article's Creative Commons licence and your intended use is not permitted by statutory regulation or exceeds the permitted use, you will need to obtain permission directly from the copyright holder. To view a copy of this licence, visit http://creativecommons.org/licenses/by/4.0/ The Creative Commons Public Domain Dedication waiver (http://creativecommons.org/publicdomain/zero/1.0/) applies to the data made available in this article, unless otherwise stated in a credit line to the data. 
(Continued from previous page)

Discussion: This study will provide evidence for a pragmatic co-operative and patient-centred educational intervention using family conferences to improve patient safety in frail elderly patients with polypharmacy.

Trial registration: German Clinical Trials Register, DRKS00015055 (WHO International Clinical Trials Registry Platform [ICTRP]). Registered on 6 February 2019.

Keywords: Frailty, Elderly patients, Polypharmacy, Family conferences, Primary care, Deprescribing, Shared decision making, Cluster randomised controlled trial, Study protocol

\section{Background}

Frailty in elderly patients is a vulnerable health status characterised by an increased risk of adverse health outcomes and/or dying when exposed to a stressor [1,2]. Physical frailty is defined as a clinical syndrome or multidimensional risk state often associated with weight loss, sarcopenia, weakness, exhaustion, and reduced physical activity $[3,4]$. Frailty is associated with an increased risk of poor health outcomes, including falls, delirium, malnutrition, hospitalisation, and mortality $[1,5]$. However, geriatric frailty is not a categorical irreversible status; it can be addressed by various interventions [6, 7].

Polypharmacy is recognised as a possible major contributor to the pathogenesis of frailty [8]. Despite the undisputable benefit of drug treatment for certain conditions, a large number of medications in patients with multimorbidity increases the risk of adverse drug reactions (ADRs) due to age-related changes in pharmacokinetics, pharmacodynamics, and physiology (resulting in higher sensitivity), as well as due to drug interactions $[9,10]$. ADRs are found in $35 \%$ of older people cared for at home [11]. It has been assumed that $2.4-6.5 \%$ of all hospital admissions are drug related. In older people, these figures are considerably higher [12-14]. Polypharmacy independently increases the risk of falling, frailty, hospital admission [15-17], and mortality [18]. Excessive polypharmacy (ten or more drugs) is an independent risk factor for death in vulnerable geriatric patients [18]. Reducing inappropriate medication exposure in frail patients is supposed to be a promising approach to improve health-related quality of life and prevent adverse outcomes [19-21].

Only very few randomised controlled trials have been conducted to investigate the outcomes of medication tapering or withdrawal of single drugs such as antihypertensives, statins, or benzodiazepines [22]. Particularly, the reduction of psychotropic drugs can often be realised with beneficial effects for patients [23]. In frail patients, drug treatment aimed at prolonging life (e.g., cardiovascular disease prevention) is often a less important therapeutic goal due to limited overall life expectancy [18], and a significant proportion of older adults is willing to reduce their number of daily drugs [24].

Selecting appropriate patients on the basis of fully informed consent from patients and carers and taking a palliative care perspective with the intention to reduce polypharmacy has been shown to be a successful process for improving older persons' quality of life [25]. A recent review summarised the effects of interventions on optimising polypharmacy in older people and found that most of the interventions were aimed at optimising surrogate outcomes such as number of drugs and number of inappropriate medications. However, the impact on patientrelevant outcomes has not been properly studied yet [26].

Many barriers to deprescribing inappropriate polypharmacy have been identified in physicians, patients, and relatives, resulting in major challenges to the communication process between the participating actors [24, 27]. Therefore, interventions to reduce polypharmacy should be embedded in a shared decision-making process of general practitioners (GPs), patients, and family carers [28]. Family carers play a pivotal role in daily drug management, such as by avoiding failure to receive drugs, paying attention to ADRs, and supporting patient adherence [29].

Family conferences are a well-established instrument in nursing, intensive care units, and palliative care [30, 31]. Family conferences are a forum for communication between patients, physicians, nursing staff, and family members [31-33]. From the families' point of view, treatment changes for a chronic illness are among the three major indications for a family conference with a GP (apart from terminal illness and hospitalisation). Although family conferences are effective in improving the communication process [31] and involvement of frail patients in planning conferences is possible [34], up to now, no intervention study has investigated their effects on polypharmacy in frail patients cared for at home.

This study evaluates the effects of a complex intervention including repeated structured medication reviews and family conferences in frail elderly patients with polypharmacy living at home. The primary hypothesis is that patient safety operationalised as the hospitalisation rate will be reduced in the intervention group compared with the control group with usual care within the study period of 12 months. Secondary hypotheses include that the intervention will reduce the number of prescribed inappropriate drugs, improve health-related quality of life, reduce the rate of falls, and reduce the rate of emergency services. Additionally, we expect that in the 
intervention group, self-determination of patients will improve and the process of shared decision making between GPs, patients, and family carers will be enhanced.

\section{Methods}

\section{Study design}

A cluster randomised controlled trial will be performed to assess the effectiveness of a single-arm complex intervention for GPs, their geriatric patients with frailty and polypharmacy living at home, and the patients' family carers. Cluster randomisation at the level of practices/ medical centres is necessary to avoid contamination because the intervention addresses a change of the professional performance of the GPs. The primary endpoint (hospitalisation rate) is measured at the patient level. Intervention/observation time per patient will be 12 months. Time between first patient in and last patient out will be approximately 15 months per GP practice and 18 months for the complete trial. The design is open label with only outcome assessors being blinded. The Consolidated Standards of Reporting Trials (CONSORT) statement (with extension for cluster trials) has been used to design the study and will be used when reporting the results.

\section{Study setting and participants}

This study will be performed in a primary care setting in two areas of Germany (Düsseldorf and Rostock). At first, 136 GPs will be recruited. The GPs will be requested to enrol 676 frail elderly patients (average of 5 patients per GP) with polypharmacy living at home (Fig. 1). Patients are eligible to participate in the study if they meet all of the following criteria:

1. Positive screening of frailty in a short screening questionnaire answered by their GP using the Canadian Study of Health and Aging Clinical Frailty Scale [5]. Patients at levels 5-7 are eligible to participate in this trial.

2. Aged 70 years or older

3. Regular intake of five or more different drugs per day (defined as polypharmacy)

4. Care dependency (need for care assessed by the medical service of the German long-term care insurance) or comparable status

5. Receiving nursing care in the domestic environment, provided either by informal family carers alone or by professional ambulatory care services

Patients are not eligible to participate in the study if they meet one or more of the following criteria as rated by their GPs:
1. Moderate or severe dementia

2. Being under legal guardianship

3. Reduced life expectancy of 6 months or less (palliative care)

4. Living in a nursing home

5. Insufficient German-language skills of patients and/ or family carer and no translator available

\section{Recruitment and incentives}

The study centres will send an invitation letter to the local GPs with a request to answer by email or fax if interested in participating in the study. In the next step, the GPs will be contacted by the study staff in order to hand out further information and to get informed consent of the GPs. In addition, some local information events on the topic of geriatric frailty syndrome will be offered to all interested GPs without disclosing the study objective, which is deprescribing. Each of the enrolled GPs is requested to include five eligible patients in the study.

Participating GPs in both the intervention group and the control group receive an expense allowance for every enrolled patient of $€ 100$. Patients and carers will not receive financial incentives for participating in the study.

\section{Intervention}

\section{Experimental intervention}

In the first phase of the Family Conferences and Shared Prioritisation to Improve Patient Safety in the Frail Elderly (COFRAIL) study, a systematic process of intervention development was conducted with collaboration of all project partners. The feasibility of the recruitment process and the COFRAIL intervention was tested in a pilot study. For this purpose, four GPs conducted eight family conferences with frail elderly patients and at least one family member. For evaluation purposes, the GPs and the family carers were interviewed by telephone after the family conferences. Conclusions from the pilot study regarding the educational concept will be drawn by consulting all project partners.

The experimental intervention will be performed in two steps:

1. Step 1: GPs allocated to the intervention arm will receive an educational intervention covering the following topics: (a) structured patient-centred medication review, (b) family conference according to a structured guideline with special focus on prioritisation of treatment goals concerning drug therapy (communication training included), and (c) structured deprescribing protocol in case of a decision in favour of reducing the drug burden. Over the course of the trial, altogether three trainings (two meetings in the first year and one optional 


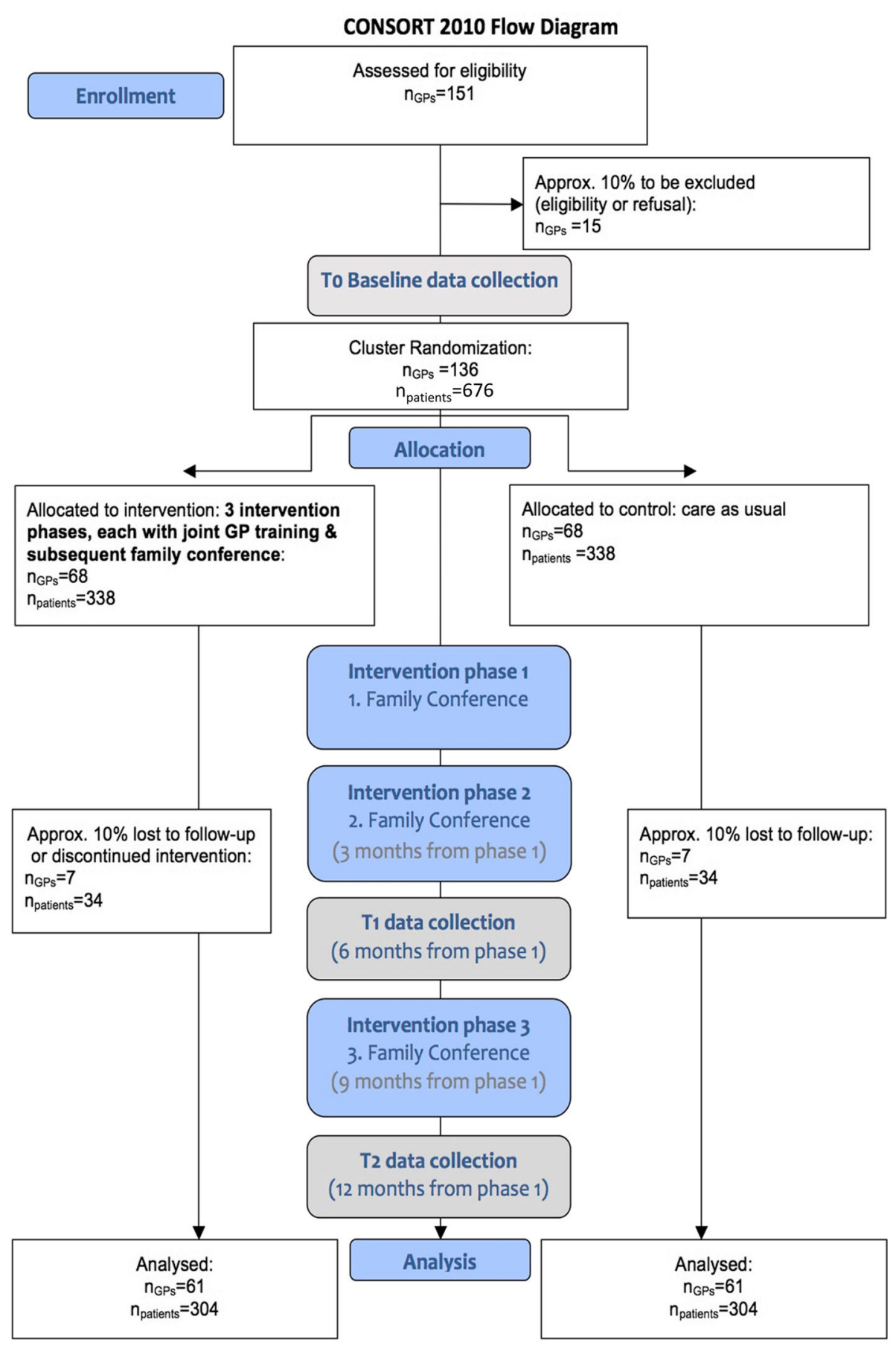

Fig. 1 Family Conferences and Shared Prioritization to Improve Patient Safety in the Frail Elderly (COFRAIL) study flow chart

meeting in the second year) are required to achieve the educational objectives and to enhance the safety of the intervention by giving the GPs the opportunity to discuss their experiences with colleagues based on their own cases. An overview of the educational intervention programme is presented in Fig. 2.
2. Step 2: During the 1-year intervention, GPs will perform a total of three family conferences per enrolled patient each, including a structured medication review with the enrolled patient and the patient's family carer instead of routine home visits or consultations. The family conferences are scheduled at the beginning of the study, after 3 months, and after 


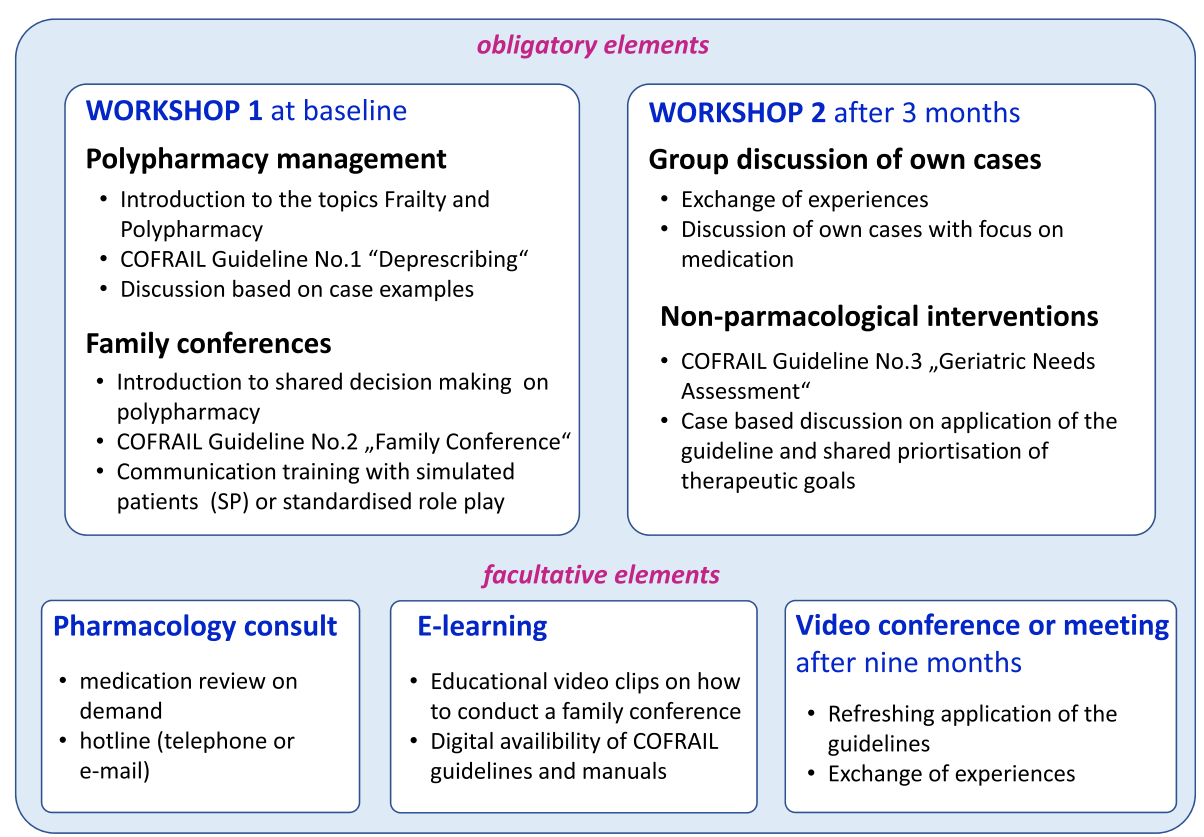

Fig. 2 Elements of the educational intervention

9 months, each lasting about 30-45 min. The conversations will initially focus on the medications taken by the patient (brown bag review), followed by a discussion about treatment goals and priorities of the patient and the family carers. The family conferences can be conducted optionally in the doctor's office or at the patient's home. If desired by the team and/or physician, counselling by an external clinical pharmacologist/pharmacist will be provided via phone or email.

If both patient and physician in agreement consider reducing polypharmacy, the GP is recommended to follow a structured deprescribing protocol based on those published by Scott [25] and Garfinkel [20], including five steps: (1) ascertain all drugs the patient is currently taking and the reasons for each one, (2) consider overall risk of drug-induced harm in individual patients to determine the required intensity of deprescribing intervention, (3) assess each drug in regard to its current or future benefit compared with current or future harm or potential burden, (4) prioritise drugs for discontinuation that have the lowest benefit-toharm ratio and lowest likelihood of adverse withdrawal reactions or disease rebound syndromes, and (5) implement a discontinuation regime and monitor patients closely for improvement in outcomes or onset of adverse effects.

In addition, the GP has the option to obtain a written medication review for the patient by a clinical pharmacologist associated with the study group.

There will be no special criteria for discontinuing or modifying allocated interventions. Implementing training and up to three family conferences may alter usual care pathways (including use of any medication) in GP practices randomised to the intervention.

There is no anticipated harm and no compensation for trial participation. It is not necessary to provide posttrial care.

\section{Control group}

Patients in the control group will receive care as usual. GPs in the control group will be offered training seminars on clinical topics for daily practice that do not interfere with the intervention.

At the end of the observational period, the educational intervention will be offered to GPs in the control group due to motivational reasons. This offer will not influence study outcomes but should maintain the motivation of the GPs assigned randomly to the control arm.

\section{Measurements}

At the beginning of the trial, the following parameters will be collected on the GP's level by a self-administered questionnaire: age, sex, number of practicing GPs in the practice, number of treatment cases per year, year of licensure to practice medicine, and board-certified specialities. The GPs will be requested to provide the following data of patients from the patients' medical records at baseline: chronic diagnoses and medication schedule. After 6 and 12 months, new chronic diagnoses and medication schedules shall be reported. This trial does not involve collecting biological specimens. 
A geriatric assessment of patients will be performed by trained study nurses during home visits at baseline, after 6 months $\left(T_{1}\right)$, and after 12 months $\left(T_{2}\right)$. The following sociodemographic parameters of patients will be recorded by study nurses: age, sex, level of education, marital status, household members, degree of disability (Grad der Behinderung), level of care dependency assessed by the medical service of the German social care insurance (Pflegegrad). In addition, the study nurse will collect data on all medications used by the patient within the last week. The data include product name, pharmaceutical form, content of the active substance, German national drug code, periodic or as needed (pro re nata) medication, dosage and frequency (for periodic medication), and duration of prescription (less than 1 week, 1 week or longer but less than 1 month, 1 month or longer but less than 2 months, 2 months or longer but less than 6 months, 6 months or longer but less than 1 year, or 1 year or longer). The interviewer will ask the patient to show the packages of the pharmaceuticals to get the most valid information.

The patients will be requested to keep an event diary to document number of falls, weight, hospitalisations, and any other health care use which is further explained below under the heading 'Additional health economic parameters'. The event diary will be handed over, collected, and validated by the study nurses during their home visits.

\section{Primary outcome}

The primary endpoint of the study is the number of hospitalisations per patient during the observation period of 12 months. This outcome does not include visits to an outpatient clinic or visits to an emergency unit without hospital admission. The hospitalisations will be documented by the participating GPs after 6 and 12 months and by the patients in their event diary. In case of disparity between the number of hospitalisations documented by GPs and patients, the study centre will be requested to validate the information on hospitalisation. If the information cannot be clarified, the higher value will be used for data analysis.

\section{Secondary outcomes}

The following secondary outcomes will be analysed on the basis of data collected by study nurses during home visits at baseline and after 6 and 12 months:

- Total number of medications, Drug Burden Index defined as the number of anticholinergic and sedative medications [16], prevalence of potentially inappropriate medications (PIMs) [35, 36], medication regimen complexity [37], and drug-drug interactions
- Weakness measured by grip strength using a dynamometer (model SH5001; Saehan Corp., Masan, Republic of Korea); best of three attempts used

- Cognition: Two short cognitive subtests from the Consortium to Establish a Registry for Alzheimer's Disease neuropsychological test battery, German version, with norms and reliable change indices recently derived from an older German GP sample [38, 39]:

Episodic memory: word list learning (three trials immediate and one trial delayed recall); total administration time: maximum of 10 min plus 10min interval and approximately $30 \mathrm{~s}$ for instruction

Executive function: semantic fluency (animal naming test) as a measure of combined verbal ability and executive control; administration time 1 min plus approximately $20 \mathrm{~s}$ for instruction

- Depressive disorder assessed by the Geriatric Depression Scale [40]

- Mobility measured by the Timed Up \& Go Test [41]

- Health-related quality of life (EQ-5D-5L) will be assessed to facilitate calculation of quality-adjusted life-years (QALYs) for use in the economic analysis [42].

- Activities of daily living measured by the Barthel index [43]

- Weight (self-reported)

- Number of falls (self-reported)

- Use of emergency services

Detailed procedures of the measurements are listed in the schedule of enrolment, interventions, and assessments (Table 1).

Participant retention and complete follow-up will be promoted via phone calls by the study nurses. For patients who drop out of the study, the following parameters are requested by the GPs: hospitalisations, cardiovascular events (e.g., stroke, myocardial infarction), mortality, and other adverse events (AEs).

\section{Additional safety parameters}

The GPs will be requested to document cardiovascular events (e.g., stroke, myocardial infarction) and mortality after 6 months $\left(\mathrm{T}_{1}\right)$ and after 12 months $\left(\mathrm{T}_{2}\right)$. The following safety parameters, if available, will be collected every 3 months by the GPs from the records:

- Blood pressure level (mmHg)

- Heart rate (beats/min)

- Laboratory results: blood sugar ( $\mathrm{mg} / \mathrm{dl})$, estimated glomerular filtration rate 


\section{Additional health economic parameters}

- Intervention costs will be collected by the study centres and include personnel expenses, costs for the educational intervention (room and catering) material costs, printing costs, and postage related to manuals for physicians and information brochures for patients

- Costs resulting from the consumption of healthrelated goods and services due to outpatient visits, visits to other health service providers, emergency admissions, hospital admissions, admissions to rehabilitation facilities, medical appliances, and support in households, and services of the long-term care insurance are estimated on the basis of patient questionnaires

\section{Sample size}

In a similar trial population, the mean rate of hospitalisations amounted to 0.38 per patient with a standard deviation of 0.75 during 6 months [44]. We assume that during an observation period of 12 months, the mean number of hospitalisations per patient will be 0.75 with a common standard deviation of 1.0, and we expect a decrease to a mean number of 0.5 in the intervention group. With a power of $80 \%$ and an alpha error of 0.05 , we would need a sample size of 253 in each group (two-sided $t$ test for equal variances) to detect the expected difference. For a cluster randomised intervention trial, the estimated sample size must be adjusted by the design effect. If an intra-cluster correlation of 0.05 is assumed and about five patients per GP are planned, the design effect would be 1.2 and the sample size would be 608 patients in 122 GP practices. With an estimated drop-out rate of $10 \%$ during the study period, 676 patients and 136 GPs will be needed in total.

\section{Randomisation}

A cluster randomisation allocating the GP practices to the intervention or control group will be performed. To ensure a balance in sample size across groups over time, block randomisation of GP practices will be used. The randomisation procedure will be provided by the data management group at Hannover Medical School after enrolment of patients. The allocation sequence is computer-generated and concealed from researchers and interviewers. Randomisation lists will be kept closed. To assure concealment of allocation, no practice can start the intervention until recruitment of patients is complete and randomisation has been performed.

\section{Data management}

Data will be entered in the local centres via an Internetbased electronic data capture system which complies with US Food and Drug Administration requirements (21 CFR part 11) and the guidelines of good clinical practice. The data will be stored in a centralised Oracle database (Oracle Corp., Redwood City, CA, USA). The data will be transferred via 128-bit SSL (Secure Sockets Layer) encryption; there will be no local storage of data. The access to the database and webserver is controlled by two consecutive firewall systems. Data will be stored with a pseudonym. The members of the study teams will have access to the electronic data entry system according to a detailed concept of roles and rights. An audit trail ensures an automatic protocol of all data entries, changes, and deletions.

Contact details (e.g., address, telephone number) of the enrolled GPs and patients will be stored separately in electronic files at the study centres in Rostock and Düsseldorf with secure access for the study staff.

\section{Data analysis}

\section{Statistical analyses}

The analysis of the primary endpoint will be conducted according to the intention-to-treat principle. Sensitivity analyses will be performed on the basis of the per-protocol population (i.e., all patients who have finished the study without any protocol violations will be included in these analyses). Missing values will not be imputed a priori. To evaluate a possible bias due to values that are not randomly missing, sensitivity analyses based on multiple imputation will be performed.

Due to cluster randomisation, hierarchic multilevel models (mixed models) with GP practice as a random effect will be applied for the analyses of primary and secondary endpoints. Possible baseline imbalances and confounding variables (e.g., age, sex, co-morbidities) will be controlled by adjustment.

For the evaluation of the primary endpoint, a linear mixed model analysis of variance will be applied.

The analyses of the secondary endpoints will be performed by using linear mixed models or mixed logistic regression models (binary or ordinal), depending of the underlying distribution of the outcome variable.

\section{Health economic evaluation}

The objective of the health economic evaluation is to determine the efficacy of the intervention by comparing cost and outcome of the intervention group with cost and outcome of the control group (care as usual). All costs associated with the intervention as well as costs resulting from the consumption of health-related goods and services [45] will be considered from the perspective of the German social insurance (statutory health insurance, long-term care insurance, and pension insurance).

In order to determine the efficacy of the intervention, a cost-effectiveness analysis in terms of additional costs per additional hospital admission averted and a cost-utility analysis, which aims to calculate the additional costs required for an improvement in QALYs, will be performed. 
While the former yields the incremental cost-effectiveness ratio (ICER), the latter estimates the incremental costutility ratio (ICUR). The QALYs are based on healthrelated quality of life, which will be measured by the EQ5D-5L and evaluated by a German tariff [46] to generate utilities. ICER and ICUR are calculated similarly as the ratio of the difference in mean costs and difference in mean outcomes between intervention and control group. Both costs and outcomes will be derived from cluster averages.

Statistical analyses will be based on the intention-totreat principle. A 95\% confidence interval will be obtained parametrically for the ICER/ICUR and nonparametrically by a bootstrap procedure [47, 48]. Univariate and probabilistic sensitivity analyses will be performed to estimate the robustness of the ICER/ICUR.

\section{Process evaluation}

To understand the change process by implementation of the intervention, a comprehensive assessment of process measures alongside the experimental study is indispensable [49]. The process evaluation follows international recommendations for process evaluation of complex interventions [50]. Therefore, different process parameters will be assessed on cluster and individual levels, respectively, with qualitative and quantitative methodological approaches (Table 2).

The recruitment procedure of clusters and patients will be documented, including documentation of the information provided on reasons for non-participation or dropout. Contextual aspects (e.g., socioeconomic and sociodemographic characteristics of physicians and patients) will be assessed at baseline.

Intervention fidelity will be determined by structured documentation for each education session by the trainers. All participants of the obligatory education sessions will be asked to complete a standardised questionnaire after the second session to evaluate the education programme. The use of the facultative educational elements will be also documented. In addition, telephone study monitoring of physicians and patients will be documented using a structured protocol.

Attitudes and experiences of the physicians related to the COFRAIL intervention, including barriers and facilitators, will be explored through four focus group interviews upon completion of the trial with a convenience sample of 6-12 participants per group. Furthermore, guidelinebased telephone interviews will be conducted in a convenience sample of ten physicians per region.

In a subgroup of 20 patient-relative dyads, experiences of families (e.g., consideration of preferences, changes in physician-patient communication, barriers and facilitators) will be assessed after 9 months (immediately after the last family conference) with separate semi-structured telephone interviews.
In addition, medication changes will be analysed for the whole study group, and differences between intervention and control groups will be compared descriptively. A detailed analysis will be performed in a subset of patients to elucidate which recommendations of the deprescribing guideline could be followed and which recommendations were either ignored or proved to be inappropriate. This procedure will allow improvement of the applicability of the guideline.

The process evaluation is purely exploratory. All quantitative data will be analysed descriptively, and the qualitative data will be analysed by content analysis [51] (Table 2).

\section{Quality assurance and safety}

Process flow and quality management of the study will be supervised quarterly by a scientific advisory board in repeated audits with study staff. The scientific advisory board will be involved in all decisions on important protocol modifications (e.g., changes to eligibility criteria, outcomes, analyses), followed by informing the relevant parties (investigators, trial participants, trial registries, journals, regulators).

During the intervention, each GP in the intervention group will be contacted at least twice by telephone to evaluate the progress of the study and to get information about potential harms for the patients regarding the safety parameters. Evidence suggests that serious AEs such as cardiovascular events (myocardial infarction, stroke) or death are not anticipated. Potential minor AEs are hyperglycaemia, elevated blood pressure, and recurrence of symptoms (e.g., dyspnoea, oedema) after stopping medication. The events will be reported to the data and safety monitoring board (DSMB) and relevant regulatory bodies as required, indicating expectedness, seriousness, severity, and causality.

To ensure high data quality, assessors (study nurses) will be trained on standardised patients regarding how to interview the patients and how to use the assessment tools. The personnel of the participating practices will not be involved in the collection of data.

Reliability training and checks will be performed before starting the study with the whole staff involved in interviewing and data collection. The quality assurance consists of procedures for prevention of insufficient data quality, detection of inaccurate or incomplete data, and action to improve data quality. In addition, the centres will regularly receive feedback by quality reports for data quality. External monitoring (e.g., for source data verification) would be desirable but too cost-intensive. As a solution, a random sample of paper crucial structure- and process-related factors will be compared with the data entries in the database.

For supervision of the study concerning the safety parameters and safety rules, a DSMB will be constituted. The DSMB will meet at least every 3 months starting and will evaluate the continuously collected safety parameters in a quarterly safety report. For this 
Table 1 Adapted SPIRIT schedule of enrolment, interventions, and assessments

\begin{tabular}{|c|c|c|c|c|c|c|c|}
\hline \multirow[b]{3}{*}{ Time point } & \multicolumn{7}{|c|}{ Study period } \\
\hline & \multirow{2}{*}{$\begin{array}{l}\text { Enrolment } \\
-\mathbf{t}_{\mathbf{1}}\end{array}$} & \multirow{2}{*}{$\begin{array}{l}\text { Baseline } \\
\mathbf{t}_{\mathbf{0}}\end{array}$} & \multicolumn{2}{|c|}{ Intervention } & \multirow[b]{2}{*}{$t_{1}$} & & \multirow{2}{*}{$\frac{\text { Close-out }}{\mathbf{t}_{\mathbf{2}}}$} \\
\hline & & & & & & & \\
\hline Month & -3 & 0 & 1 & 3 & 6 & 9 & 12 \\
\hline \multicolumn{8}{|l|}{ Enrolment } \\
\hline Eligibility screen & $x$ & & & & & & \\
\hline Informed consent & $x$ & & & & & & \\
\hline Allocation & & $x$ & & & & & \\
\hline \multicolumn{8}{|l|}{ Interventions } \\
\hline Intervention group (family conferences) & & & $x$ & $x$ & & $x$ & \\
\hline \multicolumn{8}{|l|}{ Control group (care as usual) } \\
\hline \multicolumn{8}{|l|}{ Assessments } \\
\hline Hospitalisation rate (NHPP) & & & & & $x$ & & $x$ \\
\hline Medication (TNM, DBI, PIM) & & $x$ & & & $x$ & & $x$ \\
\hline Grip strength & & $x$ & & & $x$ & & $x$ \\
\hline Cognition (CERAD) & & $x$ & & & $x$ & & $x$ \\
\hline Depression (GDS) & & $x$ & & & $x$ & & $x$ \\
\hline Mobility (Timed Up \& Go Test) & & $x$ & & & $x$ & & $x$ \\
\hline Health-related quality of life (EQ-5D-5L) & & $x$ & & & $x$ & & $x$ \\
\hline Activities of daily living & & $x$ & & & $x$ & & $x$ \\
\hline Weight & & $x$ & & & $x$ & & $x$ \\
\hline Number of falls & & $x$ & & & $x$ & & $x$ \\
\hline Use of emergency services & & $x$ & & & $x$ & & $x$ \\
\hline
\end{tabular}

Abbreviations: CERAD Consortium to Establish a Registry for Alzheimer's Disease, DBI Drug Burden Index, GDS Geriatric Depression Scale, NHPP number of hospitalisations per patient, PIM potentially inappropriate medications, SPIRIT Standard Protocol Items: Recommendations for Interventional Trials, TNM total number of medications

panel, at least two clinical and/or pharmacological researchers will be recruited who are not part of the COFRAIL project team or advisory board.

If the hospitalisation rate in the intervention group or in the control group will exceed more than $100 \%$ in comparison to the other group, the DSMB will inform the primary investigator. The primary investigator shall be responsible to consider further action to assure the safety of the study patients in consensus with all project partners and the DSMB.

\section{Dissemination policy}

The results of the study will be published in brief reports on the website of the project and in user-friendly journals for GPs and other health professionals. For scientific dissemination, the study results will be presented at national and international scientific conferences on health care research, general practice, and clinical pharmacology. We will publish the obtained scientific findings of the trial preferably in open-access journals.

\section{Discussion}

This study will provide evidence for a co-operative and patient-centred educational intervention using family conferences to improve patient safety in frail elderly patients with polypharmacy.

To our knowledge, this is the first randomised controlled trial aiming to assess the effects of family conferences on the safety of patients with geriatric frailty and polypharmacy. The intention of this project is to contribute to the complex process of deprescribing that is often characterised by vague fears of legal consequences and unclear responsibilities of the encountered actors. The strength of this study is that it will be conducted in daily clinical practice.

In case of a positive evaluation of our intervention, transfer and implementation to the German health care system could be realised in three steps. First, applicable recommendations for performing regular family conferences in patients with frailty and polypharmacy should be added to future clinical guidelines on management of multimorbidity and polypharmacy. Second, German statutory health insurance might consider paying GPs for conducting family conferences in usual care or within the limits of selective contracts. Third, family conferences may be an eligible part of a future disease management programme on multimorbidity and polypharmacy. As this 
Table 2 Elements of the process evaluation

\begin{tabular}{|c|c|c|}
\hline Focus & Documentation/assessment & Measurement point \\
\hline Feasibility of the intervention & $\begin{array}{l}\text { Piloting of family conferences } \\
\text { with two physicians/region: } \\
\text { semi-structured telephone } \\
\text { interview with physicians; } \\
\text { semi-structured telephone } \\
\text { interview with patients and } \\
\text { relatives }\end{array}$ & Piloting, prior $\mathrm{T}_{0}$ \\
\hline $\begin{array}{l}\text { Recruitment procedure of } \\
\text { physicians and patients }\end{array}$ & Protocol/region & $\mathrm{T}_{0}$ \\
\hline $\begin{array}{l}\text { Reasons for non-participation } \\
\text { or drop-out }\end{array}$ & $\begin{array}{l}\text { Structured inquiry and } \\
\text { documentation of reasons }\end{array}$ & $\mathrm{T}_{0}-\mathrm{T}_{2}$ \\
\hline $\begin{array}{l}\text { Description of crucial structure- } \\
\text { and process-related factors } \\
\text { (CRF) on cluster and patient level }\end{array}$ & $\begin{array}{l}\text { CRF-baseline data/cluster } \\
\text { and patient }\end{array}$ & $\mathrm{T}_{0}$ \\
\hline Conveyance of the intervention & $\begin{array}{l}\text { Mandatory educational } \\
\text { sessions: structured protocol } \\
\text { of each educational session. } \\
\text { Use of facultative educational } \\
\text { session: standardised } \\
\text { documentation } \\
\text { Use of individual medication } \\
\text { reviews in intervention and } \\
\text { control group: } \\
\text { standardised documentation }\end{array}$ & $\begin{array}{l}\mathrm{T}_{0} \text { (immediately after the } \\
\text { educational intervention) } \\
\mathrm{T}_{0} \\
\mathrm{~T}_{0}-\mathrm{T}_{2}\end{array}$ \\
\hline $\begin{array}{l}\text { Evaluation of telephone study } \\
\text { monitoring of physicians and } \\
\text { patients }\end{array}$ & Structured protocol & $T_{0}-T_{2}$ \\
\hline Evaluation of training & $\begin{array}{l}\text { All participants of the education } \\
\text { programme: Standardised } \\
\text { questionnaire } \rightarrow \text { evaluation } \\
\text { of the programme } \\
\text { - Attitudes } \\
\text { - Acceptance } \\
\text { - Self-efficacy } \\
\text { - Expectations }\end{array}$ & $\begin{array}{l}\mathrm{T}_{0} \text { (after the second } \\
\text { education sessions) }\end{array}$ \\
\hline Application of training content & $\begin{array}{l}\text { All physicians: semi-structured } \\
\text { protocols evaluation of family } \\
\text { conferences } \\
\text { - Acceptance } \\
\text { - Contents } \\
\text { - Duration } \\
\text { - Practicability } \\
\text { - Need for change }\end{array}$ & $\begin{array}{l}\mathrm{T}_{0,} \text { after } 3 \text { and } 9 \text { months } \\
\text { (immediately after family } \\
\text { conferences) }\end{array}$ \\
\hline $\begin{array}{l}\text { Experiences of physicians (e.g., } \\
\text { attitudes regarding intervention; } \\
\text { changes in physician-patient } \\
\text { communication; barriers and } \\
\text { facilitators) }\end{array}$ & $\begin{array}{l}\text { Four focus groups: two/region } \\
\text { with } 6 \text { to } 12 \text { physicians } \\
\text { Guideline-based telephone } \\
\text { interviews: } \\
\text { convenience sample of ten } \\
\text { physicians/region }\end{array}$ & $\begin{array}{l}\mathrm{T}_{2} \\
\mathrm{~T}_{2}\end{array}$ \\
\hline $\begin{array}{l}\text { Experiences of families (e.g., } \\
\text { consideration of preferences; } \\
\text { changes in physician-patient } \\
\text { communication; barriers and } \\
\text { facilitators) }\end{array}$ & $\begin{array}{l}\text { Guideline-based telephone } \\
\text { interviews: ten patient-relative } \\
\text { dyads/region }\end{array}$ & $\begin{array}{l}\text { After } 9 \text { months } \\
\text { (immediately after the } \\
\text { last family conference) }\end{array}$ \\
\hline
\end{tabular}

project is funded by the Innovationsfonds of the German Federal Joint Committee aiming only at projects with a high potential for implementation in the German health care system, there is a realistic chance for it, given a positive outcome of the trial.

\section{Trial status}

Patient recruitment opened on 15 March 2019 and is expected to continue to 31 March 2020. Protocol version number 1.1 (15 February 2020).

\section{Abbreviations}

ADR: Adverse drug reaction; AE: Adverse event; CERAD: Consortium to Establish a Registry for Alzheimer's Disease; COFRAll: Family Conferences and Shared Prioritisation to Improve Patient Safety in the Frail Elderly; CRF: Crucial structureand process-related factors; DBI: Drug Burden Index; DSMB: Data and safety monitoring board; GDS: Geriatric Depression Scale; GP: General practitioner; ICER: Incremental cost-effectiveness ratio; ICUR: Incremental cost-utility ratio; NHPP: Number of hospitalisations per patient; PIM: Potentially inappropriate medications; QALY: Quality-adjusted life-year; TNM: Total number of medications

\section{Acknowledgements}

We are very grateful to all participating GPs, patients, and family members. We express our gratitude to the members of the COFRAIL advisory board: 
Johanna Bensch, Madaida Lemke (AOK Nordost), Henrik Wiegelmann (selfhelp-organisation 'Wir pflegen'), Marjan van den Akker (Institute of General Practice, Johann Wolfgang Goethe University, Frankfurt am Main), Christoph Ostgathe (Palliative Care Department, University Hospital Erlangen, FriedrichAlexander-University Erlangen-Nürnberg), Vera Kalitzkus (Institute of General Practice, Medical Faculty, Heinrich-Heine-University Düsseldorf).

The members of the COFRAIL study group are as follows: Stefan Wilm (principal investigator $[\mathrm{PI}])$, Achim Mortsiefer (PI), Sara Santos, Susanne Löscher (Institute for General Practice, Centre for Health and Society, Medical Faculty, Heinrich-Heine-University Düsseldorf); Attila Altiner, Anja Wollny, Eva Drewelow, Manuela Ritzke (Institute of General Practice at the University Medical Centre Rostock); Petra Thürmann, Sven Schmiedl, Nina-Kristin Mann, Veronika Bencheva (Department of Clinical Pharmacology, School of Medicine, Faculty of Health, Witten/Herdecke University); Gabriele Meyer, Steffen Fleischer, Jens Abraham (Institute for Health and Nursing Science, Medical Faculty, Martin Luther University Halle-Wittenberg); Andrea Icks, Markus Vomhof, Joseph Montalbo (Institute for Health Services Research and Health Economics, Centre for Health and Society, Faculty of Medicine, Heinrich-HeineUniversity Düsseldorf); Birgitt Wiese (WG Medical Statistics and ITInfrastructure, Institute of General Practice, Hannover Medical School).

\section{Authors' contributions}

AM had the initial idea for the project. AM, SW, AA, PT, BW, Al, and GM initiated the study design and received a grant for the study. BW provided biostatistical expertise, including power calculation, randomisation, data entry management, and the protocol of the statistical analyses. SS, SL, AW, ED, MR, $\mathrm{NM}, \mathrm{JA}$, and JM contributed to the development of measurement methods and in drafting the manuscript. The development of the educational intervention was conducted with contributions of all members of the COFRAIL study group. All authors approved the final manuscript. All named authors adhere to the authorship guidelines of Trials. All authors have agreed to publication. No professional writers have been involved.

\section{Funding}

The study is funded by Innovationsfonds of the German Federal Joint Committee, Gutenbergstraße 13, 10587 Berlin, funding no. 01VSF17053. The sponsor played no part in study design; collection, management, analysis, or interpretation of data; writing of the report; or the decision to submit the report for publication.

\section{Availability of data and materials}

Data cannot be made available, owing to the European Union General Data Protection Regulation. Readers who are interested in study materials (e.g. model consent form) should contact the corresponding author.

\section{Ethics approval and consent to participate}

This study has been approved by the ethics committees of the study centres Rostock (no. A2018-0151) and Düsseldorf (no. 2018-283) before recruitment of GPs and patients.

In accordance with the ethical standards and the Declaration of Helsinki, written informed consent to participate in the study is obtained from all participants.

On the consent form, participants will be asked if they agree to use of their data should they choose to withdraw from the trial. Participants will also be asked for permission for the research team to share relevant data with people from the universities taking part in the research or from regulatory authorities, when relevant. This trial does not involve collecting biological specimens for storage.

\section{Consent for publication}

Not applicable.

\section{Competing interests}

The authors declare that they have no competing interests.

\section{Author details}

${ }^{1}$ Institute of General Practice, Medical Faculty, Heinrich-Heine-University, Moorenstr. 5, 40225 Düsseldorf, Germany. ${ }^{2}$ Institute of General Practice, University Medical Center Rostock, Doberaner Str. 142, 18057 Rostock, Germany. ${ }^{3}$ Department of Clinical Pharmacology, School of Medicine, Faculty of Health, Witten/Herdecke University, Heusnerstr. 40, 42283 Wuppertal,
Germany. ${ }^{4}$ Institute for Health and Nursing Science, Medical Faculty, Martin Luther University Halle-Wittenberg, Magdeburger Str. 8, 06112 Halle (Saale), Germany. ${ }^{5}$ Institute for Health Services and Economics, Centre for Health and Society, Faculty of Medicine, Heinrich-Heine-University Düsseldorf, Moorenstr. 5, 40225 Düsseldorf, Germany. ${ }^{6}$ WG Medical Statistics and IT-Infrastructure, Institute of General Practice, Hannover Medical School, Carl-Neuberg-Str. 1, 30625 Hannover, Germany.

Received: 5 January 2020 Accepted: 18 February 2020

Published online: 20 March 2020

\section{References}

1. Fried LP, Tangen CM, Walston J, Newman AB, Hirsch C, Gottdiener J, et al. Frailty in older adults: evidence for a phenotype. J Gerontol A Biol Sci Med Sci. 2001;56(3):M146-56.

2. Morley JE, Vellas B, van Kan GA, Anker SD, Bauer JM, Bernabei R, et al. Frailty consensus: a call to action. J Am Med Dir Assoc. 2013;14(6):392-7.

3. Bergman H, Ferrucci L, Guralnik J, Hogan DB, Hummel S, Karunananthan S, et al. Frailty: an emerging research and clinical paradigm-issues and controversies. J Gerontol A Biol Sci Med Sci. 2007:62(7):731-7.

4. Clegg A, Young J, lliffe S, Rikkert MO, Rockwood K. Frailty in elderly people. Lancet. 2013;381(9868):752-62.

5. Rockwood K, Song X, MacKnight C, Bergman H, Hogan DB, McDowell I, et al. A global clinical measure of fitness and frailty in elderly people. CMAJ. 2005;173(5):489-95.

6. Buckinx F, Rolland Y, Reginster JY, Ricour C, Petermans J, Bruyere O. Burden of frailty in the elderly population: perspectives for a public health challenge. Arch Public Health. 2015;73:19.

7. Cameron ID, Fairhall N, Langron C, Lockwood K, Monaghan N, Aggar C, et al. A multifactorial interdisciplinary intervention reduces frailty in older people: randomized trial. BMC Med. 2013;11:65.

8. Flaherty JH, Perry HM 3rd, Lynchard GS, Morley JE. Polypharmacy and hospitalization among older home care patients. J Gerontol A Biol Sci Med Sci. 2000;55(10):M554-9.

9. Hamilton HJ, Gallagher PF, O'Mahony D. Inappropriate prescribing and adverse drug events in older people. BMC Geriatr. 2009;9:5.

10. Reeve E, Wiese MD, Mangoni AA. Alterations in drug disposition in older adults. Expert Opin Drug Metab Toxicol. 2015;11(4):491-508.

11. Hanlon JT, Schmader KE, Koronkowski MJ, Weinberger M, Landsman PB, Samsa GP, et al. Adverse drug events in high risk older outpatients. J Am Geriatr Soc. 1997;45(8):945-8.

12. Lazarou J, Pomeranz BH, Corey PN. Incidence of adverse drug reactions in hospitalized patients: a meta-analysis of prospective studies. JAMA. 1998; 279(15):1200-5.

13. Onder G, Pedone C, Landi F, Cesari M, Della Vedova C, Bernabei R, et al. Adverse drug reactions as cause of hospital admissions: results from the Italian Group of Pharmacoepidemiology in the Elderly (GIFA). J Am Geriatr Soc. 2002;50(12):1962-8.

14. Pirmohamed M, James S, Meakin S, Green C, Scott AK, Walley TJ, et al. Adverse drug reactions as cause of admission to hospital: prospective analysis of 18820 patients. BMJ. 2004;329(7456):15-9.

15. Gnjidic D, Hilmer SN, Blyth FM, Naganathan V, Waite L, Seibel MJ, et al. Polypharmacy cutoff and outcomes: five or more medicines were used to identify community-dwelling older men at risk of different adverse outcomes. J Clin Epidemiol. 2012;65(9):989-95.

16. Hilmer SN, Mager DE, Simonsick EM, Ling SM, Windham BG, Harris TB, et al. Drug Burden Index score and functional decline in older people. Am J Med. 2009;122(12):1142-9 e1-2.

17. Fried TR, O'Leary J, Towle V, Goldstein MK, Trentalange M, Martin DK. Health outcomes associated with polypharmacy in community-dwelling older adults: a systematic review. J Am Geriatr Soc. 2014;62(12):2261-72.

18. Hubbard RE, O'Mahony MS, Woodhouse KW. Medication prescribing in frail older people. Eur J Clin Pharmacol. 2013;69(3):319-26.

19. Gnjidic D, Le Couteur DG, Kouladjian L, Hilmer SN. Deprescribing trials: methods to reduce polypharmacy and the impact on prescribing and clinical outcomes. Clin Geriatr Med. 2012;28(2):237-53.

20. Garfinkel D, Ilhan B, Bahat G. Routine deprescribing of chronic medications to combat polypharmacy. Ther Adv Drug Saf. 2015;6(6):212-33.

21. Loganathan $M$, Singh S, Franklin BD, Bottle A, Majeed A. Interventions to optimise prescribing in care homes: systematic review. Age Ageing. 2011; 40(2):150-62. 
22. Iyer S, Naganathan V, McLachlan AJ, Le Couteur DG. Medication withdrawal trials in people aged 65 years and older: a systematic review. Drugs Aging. 2008;25(12):1021-31

23. Declercq T, Petrovic M, Azermai M, Vander Stichele R, De Sutter Al, van Drie $M L$, et al. Withdrawal versus continuation of chronic antipsychotic drugs for behavioural and psychological symptoms in older people with dementia. Cochrane Database Syst Rev. 2013;3:CD007726.

24. Reeve E, Wiese MD, Hendrix I, Roberts MS, Shakib S. People's attitudes, beliefs, and experiences regarding polypharmacy and willingness to deprescribe. J Am Geriatr Soc. 2013;61(9):1508-14.

25. Scott IA, Hilmer SN, Reeve E, Potter K, Le Couteur D, Rigby D, et al. Reducing inappropriate polypharmacy: the process of deprescribing. JAMA Intern Med. 2015;175(5):827-34.

26. Cooper JA, Cadogan CA, Patterson SM, Kerse N, Bradley MC, Ryan C, et al. Interventions to improve the appropriate use of polypharmacy in older people: a Cochrane systematic review. BMJ Open. 2015;5(12):e009235.

27. Anderson K, Stowasser D, Freeman C, Scott I. Prescriber barriers and enablers to minimising potentially inappropriate medications in adults: a systematic review and thematic synthesis. BMJ Open. 2014;4(12):e006544.

28. Bokhof B, Junius-Walker U. Reducing polypharmacy from the perspectives of general practitioners and older patients: a synthesis of qualitative studies. Drugs Aging. 2016;33(4):249-66.

29. Travis SS, Bernard MA, McAuley WJ, Thornton M, Kole T. Development of the family caregiver medication administration hassles scale. Gerontologist. 2003:43(3):360-8.

30. Singer AE, Ash T, Ochotorena C, Lorenz KA, Chong K, Shreve ST, et al. A systematic review of family meeting tools in palliative and intensive care settings. Am J Hosp Palliat Care. 2016;33(8):797-806.

31. Powazki R, Walsh D, Hauser K, Davis MP. Communication in palliative medicine: a clinical review of family conferences. J Palliat Med. 2014;17(10): 1167-77.

32. Kushner K, Meyer D. Family physicians' perceptions of the family conference. J Family Pract. 1989;28(1):65-8.

33. Comley A. Family therapy and the family physician. Can Fam Physician. 1973;19(2):78-81.

34. Bangsbo A, Duner A, Liden E. Patient participation in discharge planning conference. Int J Integr Care. 2014;14:e030.

35. Holt S, Schmiedl S, Thürmann PA. Potentially inappropriate medications in the elderly: the PRISCUS list. Dtsch Arztebl Int. 2010;107(31-32):543-51.

36. Renom-Guiteras A, Meyer G, Thürmann PA. The EU (7)-PIM list: a list of potentially inappropriate medications for older people consented by experts from seven European countries. Eur J Clin Pharmacol. 2015;71(7): 861-75

37. George J, Phun YT, Bailey MJ, Kong DC, Stewart K. Development and validation of the medication regimen complexity index. Ann Pharmacother. 2004;38(9):1369-76.

38. Luck T, Riedel-Heller SG, Wiese B, Stein J, Weyerer S, Werle J, et al. CERADNP battery: age-, gender- and education-specific reference values for selected subtests: results of the German Study on Ageing, Cognition and Dementia in Primary Care Patients (AgeCoDe) [in German]. Z Gerontol Geriatr. 2009:42(5):372-84.

39. Stein J, Luppa M, Maier W, Tebarth F, Heser K, Scherer M, et al. The assessment of changes in cognitive functioning in the elderly: age- and education-specific reliable change indices for the SIDAM. Dement Geriatr Cogn Disord. 2012;33(2-3):73-83.

40. Yesavage JA, Brink TL, Rose TL, Lum O, Huang V, Adey M, et al. Development and validation of a geriatric depression screening scale: a preliminary report. J Psychiatr Res. 1982;17(1):37-49.

41. Podsiadlo D, Richardson S. The Timed 'Up \& Go': a test of basic functional mobility for frail elderly persons. J Am Geriatr Soc. 1991;39(2):142-8.

42. Herdman M, Gudex C, Lloyd A, Janssen M, Kind P, Parkin D, et al. Development and preliminary testing of the new five-level version of EQ-5D (EQ-5D-5L). Qual Life Res. 2011;20(10):1727-36.

43. Wade DT, Collin C. The Barthel ADL Index: a standard measure of physical disability? Int Disabil Stud. 1988;10(2):64-7.

44. Hajek A, Brettschneider C, Lange C, Posselt T, Wiese B, Steinmann S, et al. Longitudinal predictors of institutionalization in old age. PLoS One. 2015; 10(12):e0144203.

45. Krauth C, Hessel F, Hansmeier T, Wasem J, Seitz R, Schweikert B. Empirical standard costs for health economic evaluation in Germany - a proposal by the working group Methods in Health Economic Evaluation [in German] Gesundheitswesen. 2005:67(10):736-46.

46. Greiner W, Claes C, Busschbach JJ, von der Schulenburg JM. Validating the EQ-5D with time trade off for the German population. Eur J Health Econ. 2005;6(2):124-30.

47. Goodman P, Mackey MC, Tavakoli AS. Factors related to childbirth satisfaction. J Adv Nurs. 2004:46(2):212-9.

48. Schöffski O. Graf von der Schulenburg JM. Gesundheitsökonomische Evaluationen. 3rd ed. Berlin, Heidelberg: Springer; 2008.

49. Craig P, Dieppe P, Macintyre S, Michie S, Nazareth I, Petticrew M, et al. Developing and evaluating complex interventions: the new Medical Research Council guidance. BMJ. 2008;337:a1655

50. Moore GF, Audrey S, Barker M, Bond L, Bonell C, Hardeman W, et al. Process evaluation of complex interventions: Medical Research Council guidance. BMJ. 2015:350:h1258.

51. Mayring P. Qualitative content analysis: basics and techniques [in German]. 11th ed. Deutscher Studien Verlag: Weinheim; 2010.

\section{Publisher's Note}

Springer Nature remains neutral with regard to jurisdictional claims in published maps and institutional affiliations.

\section{Ready to submit your research? Choose BMC and benefit from:}

- fast, convenient online submission

- thorough peer review by experienced researchers in your field

- rapid publication on acceptance

- support for research data, including large and complex data types

- gold Open Access which fosters wider collaboration and increased citations

- maximum visibility for your research: over $100 \mathrm{M}$ website views per year

At BMC, research is always in progress.

Learn more biomedcentral.com/submissions 